\title{
A Missed Differential in an Extra-Axial Lesion with Calvarial Involvement
}

\author{
Madhivanan Karthigeyan ${ }^{a}$ Prankul Singhal $^{a}$ Pravin Salunke $^{a} \quad$ Kirti Gupta $^{b}$ \\ ${ }^{a}$ Department of Neurosurgery, and ${ }^{b}$ Department of Histopathology, Postgraduate Institute of Medical Education \\ and Research (PGIMER), Chandigarh, India
}

\section{Keywords}

Supratentorial ependymoma - Extra-axial ependymoma .

Anaplastic ependymoma $\cdot$ Ependymoma $\cdot$ Meningioma

\begin{abstract}
Extra-axial central nervous system lesions can occasionally involve the overlying calvarium. The involvement may be due to longstanding pressure effects or secondary to the aggressive behaviour of the lesion. The spectrum of such duralbased lesions varies from infectious/inflammatory to neoplastic etiology. We present a dural-based lesion with the erosion of the overlying calvarium in a young lady. In view of the short history with heterogenous enhancement on magnetic resonance imaging and bony erosion, hemangiopericytoma or malignant meningioma was suspected. The histopathology, however, proved it to be an anaplastic (grade III) ependymoma. Extra-axial ependymomas though uncommon have been reported. However, these extra-axial ependymomas presenting with calvarial erosion are unknown. The bony erosion, as in the present case, may suggest an aggres-
\end{abstract}

\section{KARGER}

(c) 2017 S. Karger AG, Base

E-Mail karger@karger.com

www.karger.com/aon sive tumour behaviour. The reported extra-axial supratentorial ependymomas along with their salient radiological/operative features are shortly reviewed. Also, the differentials of extra-axial lesions with calvarial involvement are touched upon.

(c) 2017 S. Karger AG, Basel

\section{Introduction}

The differential diagnoses of an extra-axial-dural based lesion with calvarial involvement are varied. The lesions include, apart from the classical benign meningiomas, certain malignant and non-neoplastic pathologies as well [1]. A few may require surgical excision, while some of them respond well only to chemotherapy/radiotherapy $[2,3]$. In literature, a few lesions were even subjected to radiosurgery presuming them to be meningiomas and other benign lesions $[4,5]$. However, they turned out to be an altogether different entity after a definitive histopathology was conducted. Thus, distinguishing
Dr. Madhivanan Karthigeyan

Assistant Professor, Department of Neurosurgery PGIMER, Sector 12

Chandigarh 160012 (India)

E-Mail karthigeyanm82@gmail.com 

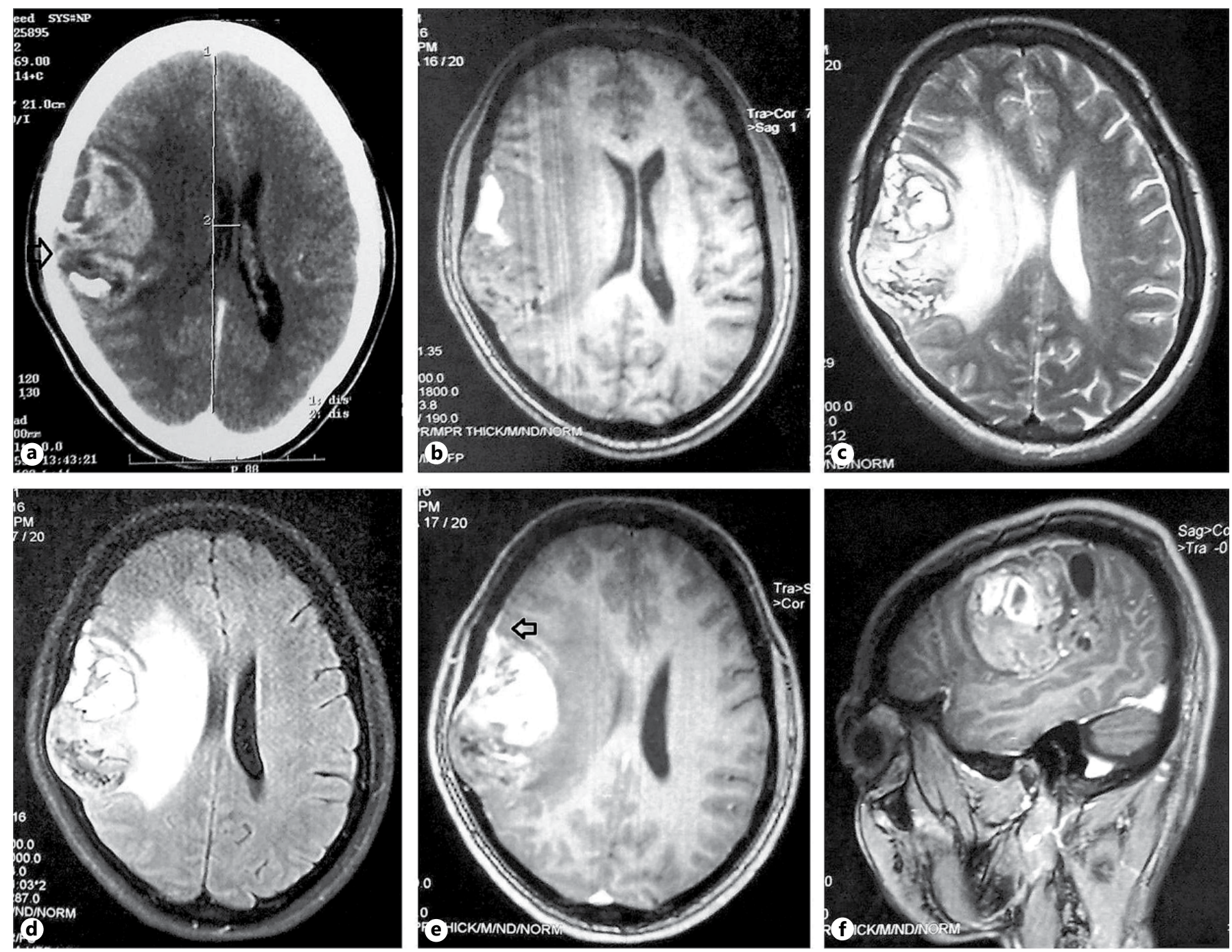

Fig. 1. a Contrast-computed tomography shows heterogenously enhancing right fronto-parietal dural-based lesion with an area of calcification. The overlying bone is irregular and thinned out (ar-

these preoperatively has a significant bearing on management.

In this study, we report an extra-axial lesion with calvarial erosion that unexpectedly proved to be an anaplastic (grade III) variety of ependymoma. Although prior reports have identified certain differentiating radiological features that suggest the presence of an ependymoma, the secondary bony changes in extra-axial ependymomas were not described $[4,6,7]$. Further, most of these have been grade II tumours $[4,6]$. The patient's clinical course, relevant literature on supratentorial extra-axial ependymomas (SEE) and the possible differential diagnosis of such lesions with calvarial involvement are briefly addressed. row); b-f T1, T2, FLAIR, and contrast images show heterogenously enhancing extra-axial mass with cystic and necrotic areas along with dural enhancement (arrow; e).

\section{Case Description}

A 33-year-old-lady presented with a 3-weeks history of progressive holocranial headache, drooping of right eyelid and diplopia. On examination, she had right partial ptosis. No other focal neurological deficits were noted. Contrast computed tomography showed heterogenously enhancing right fronto-parietal extra-axial dural-based lesion with focal areas of hypodensity and calcification (Fig. 1a). There was perilesional oedema with midline shift and mass effect over adjacent brain. The bone overlying the tumour was partly thinned out. On Gadolinium-enhanced magnetic resonance (MR) imaging, the lesion was T1 iso to hyperintense, T2 and FLAIR hyperintense and showed heterogenous enhancement with dural tail (Fig. 1b-f). There were also small necrotic and cystic areas. The differentials considered were an atypical meningioma, gliosarcoma and hemangiopericytoma. 


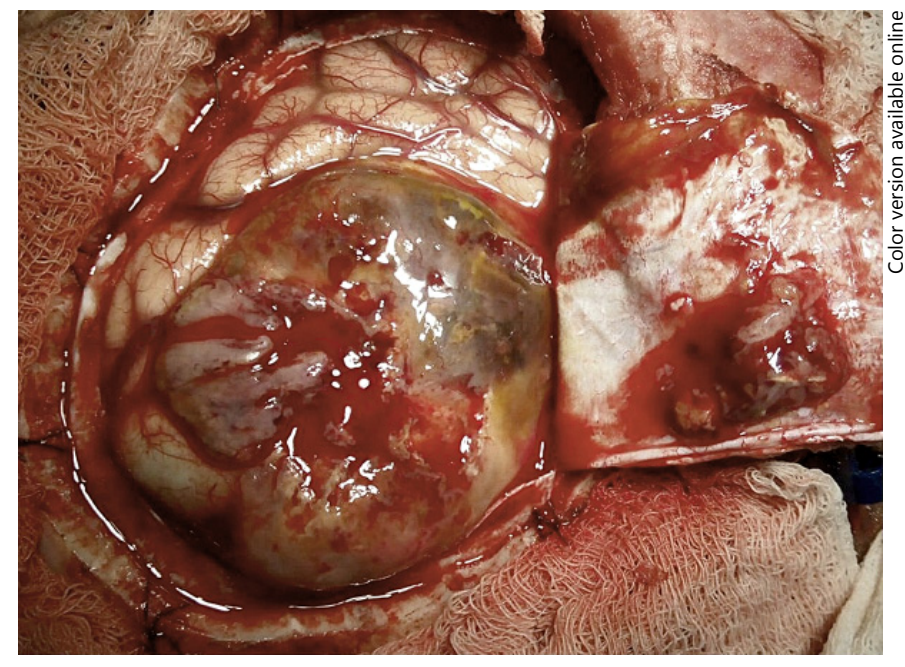

Fig. 2. Intraoperative photograph shows an extra-axial tumour that is partly attached to the inner surface of the duramater.

At surgery, the bone overlying the lesion was eroded and thinned out. The external dural surface was intact. On durotomy, the tumour was partly attached to the inner surface of dura (Fig. 2). It was soft, suckable and highly vascular with areas of necrosis. There was a well-delineated plane of cleavage with the surrounding brain except for a small area where the pial layer was involved. Gross total excision of the tumour was achieved.

Histopathology and immunohistochemical studies showed it to be an anaplastic ependymoma (WHO grade III, 2007; Fig. 3). The patient underwent adjuvant radiotherapy and was asymptomatic at 1-year follow-up.

\section{Discussion}

In adults, ependymomas are infrequent and occur in the supratentorial region adjacent to ventricles. Their extra-axial location is uncommon [4, 6-8]. Though histologically similar, the tumour biology and clinical behaviour of these differ from their infratentorial and spinal counterparts. Complete safe tumour resection is advised in SEE as in any other case of ependymoma $[4,6]$. Adjuvant radiotherapy is recommended for anaplastic tumours; the role of chemotherapy is limited $[4,6]$.

To date, 20 cases of intracranial extra-axial ependymomas have been reported $[4,6-8]$. Twelve ( 8 adults and 4 children) of them were SEE. The tumours have varying (iso to hyper) T1, T2 MR signal intensities, with the enhancement pattern being heterogenous (occasionally homogenous) with some areas showing cystic changes and calcification. None had the dural tail sign that is characteristically seen in meningiomas. In a majority of cases, the preoperative diagnosis was meningioma and some were thought to be gliomas. Most tumours had good brain-tumour interface at surgery. Some had dural attachment and others did not. The most common histopathology reported was grade II. To date, only 3 cases of adult anaplastic ependymomas located extra-axially have been reported $[4,7,8]$.

There had been many instances in which an extra-axial lesion radiologically labelled as meningioma turned out to be an ependymoma on histopathology [4, 6-8]. Even in our patient, the primary diagnosis was an atypical meningioma and a possibility of the presence of ependymoma was never considered. Ours was a dural-based SEE with no cortical or ventricular connection. A unique additional feature that was noted was the overlying bony change that has not been described earlier. It is presumable that longstanding intracranial lesions may cause pressure effects and secondary changes in calvarium. However, in our patient with a short clinical history of few weeks, the calvarial erosion and thinning could be possibly attributed to the aggressive behaviour of the tumour.

Considering the rising number of reports, ependymoma in supratentorial extra-axial lesions should be kept as a differential especially in the absence of typical imaging of meningiomas. Whenever in doubt, additional sequence such as MR spectroscopy might be helpful. Ependymoma shows myo-inositol/glycine peaks, whereas alanine and glutamate/glutamine peaks are seen with meningiomas [4]. The heterogenous enhancement pattern with cystic and calcific changes in a supratentorial extraaxial tumour along with MR peaks (myo-inositol/glycine), with absent classical dural tail of meningioma may provide a clue towards the diagnosis of ependymoma. Overlying bone thinning and erosion as observed in the present case may suggest the presence of ependymoma, which is in contradistinction to the classical hyperostosis of meningioma. Occasionally, meningiomas can cause a mixed (osteoblastic and lytic) picture. However, pure lytic lesions are very rare.

There are a large number of extra-axial lesions that are frequently confused with the commonly encountered meningiomas $[1,9]$. A few of them show associated focal calvarial erosion. The possible lesions with such presentation are hemangiopericytoma, metastasis, solitary fibrous tumour, plasmacytoma, Ewing's sarcoma, lymphoma and tuberculoma. They could be either a primarily extraaxial lesion with secondary calvarial changes or a bony lesion with predominant extra-axial soft tissue component. The latter presentation, though less common, may occur with primary calvarial plasmacytoma, Ewing's sar- 

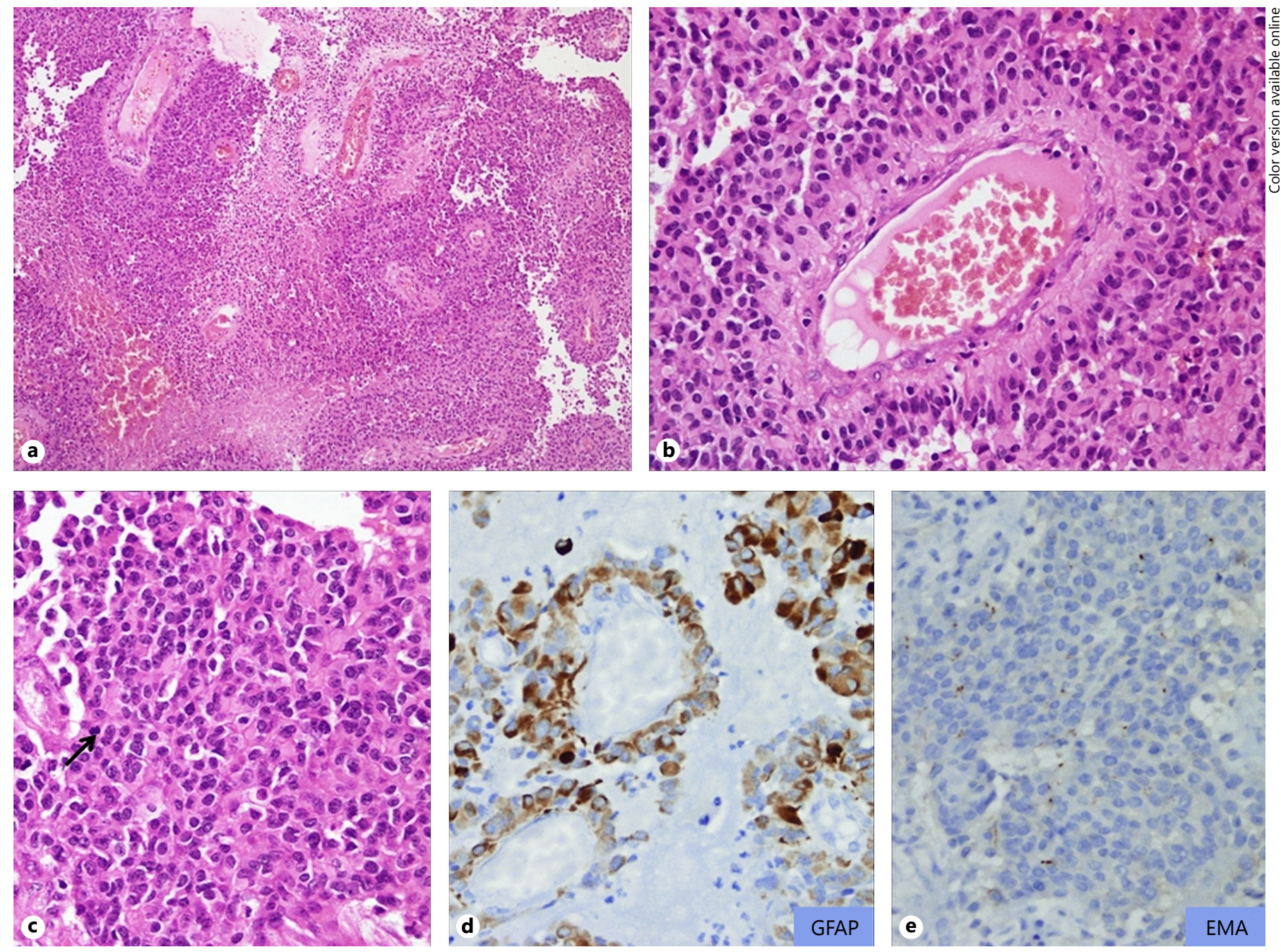

Fig. 3. a Tumour cells arranged in sheets and in perivascular location $(H \& E \times 100)$. b High magnification depicting the arrangement of cells around vessels forming pseudopapillae. No well-defined pseudo rosettes were identified $(\mathrm{H} \& \mathrm{E} \times 400)$. c Round to oval tumour cells with evenly dispersed chromatin. Mitotic figures were identified (arrow) with areas of necrosis

coma and lymphoma, which show excellent chemo/radio sensitivity. Hence, a reliable preoperative identification of such lesions could perhaps avoid unwarranted surgery at least in some patients. Fine needle aspiration cytology would suffice as the first-line investigation method in these cases. Again, the need for tissue diagnosis before attempting radiosurgery in small extra-axial lesions resembling meningiomas has been emphasized earlier by authors $[4,6,7]$. Hence, we feel it may not be wiser to subject patients to radiosurgery solely based on radiological appearance until other similar meningioma-mimicking etiologies are reasonably excluded.
$(\mathrm{H} \& \mathrm{E} \times 400)$. d Glial fibrillary acidic protein (GFAP) immunoreactivity within tumour cells with perivascular accentuation (immunoperoxidase $\times 400$ ). e Epithelial membrane antigen (EMA) demonstrates focal dot-like immunoreactivity (immunoperoxidase $\times 400$ ).

Certain salient imaging features aid in the differentiation of extra-axial bone involving lesions. Hemangiopericytoma differ from meningioma in that they show frequent bone erosion, no intralesional calcification, narrow dural attachment and heterogenous enhancement as opposed to the hyperostosis, presence of calcifications and broad dural attachment of meningiomas [1]. Primary dural and cranial vault lymphomas can have an MR picture similar to that of a meningioma. However, the underlying vasogenic brain oedema and the absence of clear-cut brain-tumour interface may suggest the presence of a lymphoma [1]. The overlying calvarium may show hyperos- 
tosis or bone erosion. Lymphomas of cranial vault display a permeative growth pattern with soft tissue mass in both extradural and galeal compartments. The intervening calvarium is lytic in $75 \%$, hyperostotic in $5 \%$ and apparently normal in $18 \%$ [3]. Calvarial metastatic lesions may be single or multiple and show significant osteolysis excepting the osteoblastic or mixed pattern seen with breast and prostate malignancies. At times, plasmacytomas present as extra-axial homogeneously enhancing soft tissue mass and involve overlying calvarium. The osteolytic lesion shows a sharp border without a sclerotic rim. Solitary fibrous tumour exhibit areas of T2 hypointensity with heterogenous signal intensity (T2 mixed hypo and hyperintense) with avid contrast enhancement and smooth bony erosion [1]. Cranial Ewing's sarcoma occurs in the first two decades, commonly involve temporal bone and present as lytic lesion with dural-based and subgaleal soft tissue mass [2]. Dural-based lesion showing T2 hypointense rim and perilesional oedema along with lipid and lactate peaks on MR spectroscopy may provide a clue towards the diagnosis of tuberculoma [9].

\section{Conclusion}

Although uncommon, ependymomas are to be considered differential of supratentorial extra-axial duralbased lesions. There may be subtle hints such as heterogenous enhancement, MR spectroscopy peaks, and calvarial thinning, which suggest the presence of an ependymoma. Calvarial erosion in the present case possibly suggests an aggressive nature of anaplastic ependymoma.

\section{Statement of Ethics}

All ethical considerations have been adhered to and informed consent was obtained from patients.

\section{Disclosure Statement}

The authors have no conflicts of interest to declare. The authors report of receiving no funding.

\section{References}

1 Smith AB, Horkanyne-Szakaly I, Schroeder JW, Rushing EJ: From the radiologic pathology archives: mass lesions of the dura: beyond meningioma-radiologic-pathologic correlation. Radiographics 2014;34:295-312.

-2 Salunke PS, Gupta K, Malik V, Kumar N, Henke LE, Cai C, et al: Primary Ewing's sarcoma of cranial bones: analysis of ten patients. Acta Neurochir (Wien) 2011;153:1477-1485.

- 3 El Asri AC, Akhaddar A, Baallal H, Boulahroud $\mathrm{O}$, Mandour $\mathrm{C}$, Chahdi $\mathrm{H}$, et al: Primary lymphoma of the cranial vault: case report and a systematic review of the literature. Acta Neurochir (Wien) 2012;154:257-265; discussion 265.
Yang Y, Tian KB, Hao SY, Wu Z, Li D, Zhang JT, et al: Primary intracranial extra-axial anaplastic ependymomas. World Neurosurg 2016;90:704.e1-e9.

5 Mukherjee KK, Salunke P: Uncontrolled spread following radiosurgery for a skull base aspergilloma misdiagnosed as schwannoma: is radiosurgery responsible? Acta Neurochir (Wien) 2016;158:643-646.

6 Salunke P, Kovai P, Sura S, Gupta K: Extraaxial ependymoma mimicking a parasagittal meningioma. J Clin Neurosci 2011;18:418420 .
Singh V, Turel MK, Chacko G, Joseph V, Rajshekhar V: Supratentorial extra-axial anaplastic ependymoma mimicking a meningioma. Neurol India 2012;60:111113.

8 Gupta VK, Kapoor I, Radotra BD, Chatterjee D: Pure extra-axial supratentorial anaplastic ependymoma with no cortical attachment: a rare case. Neurol India 2016;64:345348

Aggarwal A, Patra DP, Gupta K, Sodhi HB: Dural tuberculoma mimicking meningioma: a clinicoradiologic review of dural en-plaque lesions. World Neurosurg 2016;88:686.e1e7. 